Old Dominion University

ODU Digital Commons

Electrical \& Computer Engineering Faculty

Publications

Electrical \& Computer Engineering

2019

\title{
Non-Contact Trapping and Stretching of Biological Cells Using Dual-Beam Optical Stretcher On Microfluidic Platform
}

Aotuo Dong

Balaadithya Uppalapati

Shariful Islam

Brandon Gibbs

Old Dominion University

Ganesan Kamatchi

See next page for additional authors

Follow this and additional works at: https://digitalcommons.odu.edu/ece_fac_pubs

Part of the Biophysics Commons, Cell Biology Commons, and the Other Analytical, Diagnostic and Therapeutic Techniques and Equipment Commons

\section{Original Publication Citation}

Aotuo Dong, Balaadithya Uppalapati, Md. Shariful Islam, Brandon Gibbs, Ganesan Kamatchi, Sacharia Albin, and Makarand Deo "Non-contact trapping and stretching of biological cells using dual-beam optical stretcher on microfluidic platform", Proc. SPIE 10972, Health Monitoring of Structural and Biological Systems XIII, 109722A (1 April 2019); https://doi.org/10.1117/12.2514299.

This Conference Paper is brought to you for free and open access by the Electrical \& Computer Engineering at ODU Digital Commons. It has been accepted for inclusion in Electrical \& Computer Engineering Faculty Publications by an authorized administrator of ODU Digital Commons. For more information, please contact digitalcommons@odu.edu. 


\section{Authors}

Aotuo Dong, Balaadithya Uppalapati, Shariful Islam, Brandon Gibbs, Ganesan Kamatchi, Sacharia Albin, and Makarand Deo 


\title{
Non-contact trapping and stretching of biological cells using dual-beam optical stretcher on microfluidic platform
}

Aotuo Dong, Norfolk State University; Balaadithya Uppalapati, Norfolk State University; Md Shariful Islam, Norfolk State University; Brandon Gibbs, Old Dominion University; Ganesan Kamatchi, Sacharia Albin, Makarand Deo, Norfolk State University

\begin{abstract}
Optical stretcher is a tool in which two counter-propagating, slightly diverging, and identical laser beams are used to trap and axially stretch microparticles in the path of light. In this work, we utilized the dual-beam optical stretcher setup to trap and stretch human embryonic kidney (HEK) cells and mammalian breast cancer (MBC) cells. Experiments were performed by exposing the HEK cells to counter-propagating laser beams for 30 seconds at powers ranging from $100 \mathrm{~mW}$ to $561 \mathrm{~mW}$. It was observed that the percentage of cell deformation increased from $16.7 \%$ at $100 \mathrm{~mW}$ to $40.5 \%$ at $561 \mathrm{~mW}$ optical power. The MBC cells exhibited significantly higher cell stretching compared to HEK cells at the same power ( $80 \mathrm{~mW})$. Moreover, the minimum trapping power in HEK cells was $80.5 \mathrm{~mW}$ as compared to $65.2 \mathrm{~mW}$ in MBC cells. This study provides useful insights into the characterization of cytoskeletal elasticity in different cell types based on non-contact optical cell stretching.
\end{abstract}

\section{INTRODUCTION}

Optical Tweezers (OT) also known as single-beam gradient force trap is a technique in which small objects are immobilized and manipulated without any mechanical contact using coherent light beams. OT has been widely used in cell biology for studying the biophysical properties of cells, analyzing the structure of DNA and cell sorting [1]. OTs utilize change in momentum of a light beam when it interacts with a particle in its path to trap it by exerting a proportional force on the particle surface. This concept of laser trapping to manipulate glass spheres and micron sized latex was reported in 1970 by Arthur Ashkin while working at Bell Labs [2]. A dual-beam optical stretcher (OS) [3], on the other hand, consists of two counter-propagating, slightly diverging, and identical laser beams to trap and deform an object in the path of beams as shown in the Figure 1.

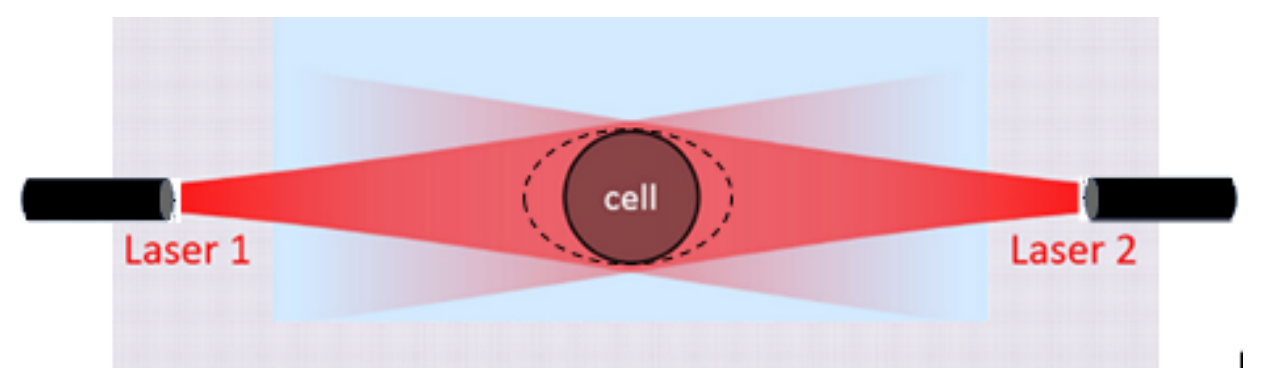

Figure 1: Principal of cell stretching in a dial-beam optical stretcher [4].

Health Monitoring of Structural and Biological Systems XIII, edited by Paul Fromme, Zhongqing Su, Proc. of SPIE Vol. 10972, 109722A · (C) 2019 SPIE

CCC code: $0277-786 X / 19 / \$ 18 \cdot$ doi: $10.1117 / 12.2514299$ 
The principle of an OS can be explained using the ray optics approach. Whenever a ray of light is reflected or refracted at an interface between media with different refractive indices, changing direction or velocity, its momentum changes. Since momentum is conserved, some momentum is transferred from the light to the interface. Due to this momentum transfer, by Newton's second law a force is exerted on the surface, which is responsible for stretching the cells. OSs have been demonstrated as a valuable tool for cell comparison between normal and cancerous cells. Guck et al. [5] exploited it to evaluate variations in the mechanical properties of various cell lines including healthy, tumorigenic and metastatic cells. It was reported that the cell membrane stiffness or cell's cytoskeletal elasticity can be effectively used as an indicator of early onset of disease such as cancer [5]. The use of OS to study the mechanical properties of cells has distinct advantages over other methods, such as, micropipette aspiration [6], micro plate manipulation [7] and Atomic force microscopy [8], all of which involve mechanical contact with the cells. OS on the other hand is a non-contact method. Therefore, chances of physical/mechanical damage to the cells being investigated are eliminated. In this study, we used a dual-beam OS setup to characterize the deformation properties of human embryonic kidney (HEK) and mammalian breast cancer (MBC) cells. Our aim was to quantify the differences in behavior of the two cell types in OS, which could be exploited in cell sorting as well as early detection of breast cancer.

\section{METHOD}

\subsection{Stress distribution}

Consider a spherical microparticle or a biological cell trapped in the path of a light beam as shown in Figure 2. By using ray optics approach, the forces acting on the surface of the cell can be estimated. The ray optics approach is valid when the size of the trapped object is much larger than the wavelength of the light $(\lambda)$. The typical diameters of biological cells such as HEK cells are in the range of $20-40 \mu \mathrm{m}$, which satisfies the ray optics criterion. i.e. $2 \pi \rho / \lambda \gg 1$, where $\rho$ is the radius of the sphere [9].

The idea is to dissociate an incident laser beam into individual rays with appropriate intensity, momentum, and direction. Each ray carries a certain amount of momentum $p$ proportional to its energy $E$ and the refractive index $n$ of the medium it travels in,

$$
p=\frac{n \cdot E}{c},
$$

where, $c$ is the speed of light in vacuum. When a ray hits the interface between two dielectric media with refractive indices $n_{1}$ and $n_{2}$, some of the ray's energy is reflected from the interface and the rest is transmitted. The reflection and transmission coefficients are calculated from Fresnel formulas [10]. The momentum of the reflected ray is

$$
p_{r}=\frac{n_{1} \cdot R \cdot E}{c}
$$


and the momentum of the transmitted ray is

$$
p_{t}=\frac{n_{2} \cdot(1-R) \cdot E}{c} .
$$

The incident momentum,

$$
p_{i}=\frac{n_{1} \cdot R \cdot E}{c},
$$

has to be conserved at the interface. The difference in momentum between the incident ray and the reflected and transmitted rays, $\Delta p=p_{i}-\left(p_{r}+p_{t}\right)$, is picked up by the surface, which experiences a force $F$ according to Newton's second law,

$$
F=\frac{\Delta p}{\Delta t}=\frac{n_{1} \cdot \Delta E}{c \cdot \Delta t}=\frac{n_{1} \cdot Q \cdot P}{c}
$$

where $P$ is the incident light power and $Q$ is a factor that describes the amount of momentum transferred. When a single light ray hits the front surface (outer wall of the cell) of the cell, it undergoes first reflection and transmission (see Figure 2). The transmitted ray inside the cell reaches the back surface (inner wall of the cell) and undergoes second reflection and transmission. Momentum transfer happens at both front and back surfaces, which implies that the surface of the cell experiences force on both the front and back surfaces. Therefore, the factor $Q$ will have two components $Q_{\text {front }}$ and $Q_{\text {back }}$, representing the momentum transfer at the front and back surfaces, respectively.

Depending on the shape of the biological cell, different analytical models are proposed to obtain the exact the $Q_{\text {front }}$ and $Q_{\text {back }}$ expressions. For instance, Guck et al [1] proposed an approximate model to obtain the expression for $Q$. This model approximates the biological cell as a cube to derive the $Q_{\text {front }}$ and $Q_{\text {back }}$ expressions for two different cases i.e. for normal and oblique incidences. Later on, Paul Bareil et al [10,11] assumed the biological cell as an exact sphere and derived more realistic formulation for $Q$.

Based on the angles defied in Figure 2, the expressions for the components of $Q$ on the front surface are

$$
\begin{aligned}
& Q_{\text {front } X}=\left[\cos (\delta)-n \cdot T(\varepsilon) \cdot \cos \left(\phi_{1}-\beta\right)+R(\varepsilon) \cdot \cos (2 \varepsilon-\delta)\right], \\
& Q_{\text {front } Y}=\left[\sin (\delta)-n \cdot T(\varepsilon) \cdot \sin \left(\phi_{1}-\beta\right)+R(\varepsilon) \cdot \sin (2 \varepsilon-\delta)\right] .
\end{aligned}
$$

Similarly for the back surface

$$
Q_{\text {backX }}=T(\varepsilon) \cdot\left[n \cdot \cos \left(\phi_{1}-\beta\right)+n \cdot R(\beta) \cdot \cos \left(3 \beta-\phi_{1}\right)+T(\beta) \cdot \cos \left(\varepsilon+\phi_{1}-2 \beta\right)\right],
$$




$$
Q_{\text {back } Y}=T(\varepsilon) \cdot\left[n \cdot \sin \left(\phi_{1}-\beta\right)+n \cdot R(\beta) \cdot \sin \left(3 \beta-\phi_{1}\right)+T(\beta) \cdot \sin \left(\varepsilon+\phi_{1}-2 \beta\right)\right] .
$$

It should be noted that, while deriving these equations the state of polarization of the light is not considered which can affect the reflectance and transmittance. By using Eqs. (6)-(9) the expression for total $Q$ is

$$
\begin{gathered}
Q_{\text {tot }}=Q_{\text {front }}+Q_{\text {back }}, \\
Q_{\text {tot }}=\sqrt{\left(Q_{\text {front } X}\left(\phi_{1}\right)\right)^{2}+\left(Q_{\text {front } Y}\left(\phi_{1}\right)\right)^{2}}+\sqrt{\left(Q_{\text {back } X}\left(\phi_{2}\right)\right)^{2}+\left(Q_{\text {back } Y}\left(\phi_{2}\right)\right)^{2}} .
\end{gathered}
$$

By using Eq. (11) we can obtain the amount of momentum transferred to each and every point on the surface of the sphere uniquely because the final momentum is a function of both $\phi_{1}$ and $\phi_{2}$. By using these polar angles every location on the sphere can be uniquely determined. Therefore by using Eq. (5), we can calculate the force acting on every element on the surface of sphere.

Once the force is obtained, the expression for surface stress or the stress acting on every surface element of the sphere is derived as

$$
\sigma=\frac{n_{1}}{c} \cdot \frac{P}{A} Q=\frac{n_{1}}{c} \cdot I \cdot Q .
$$

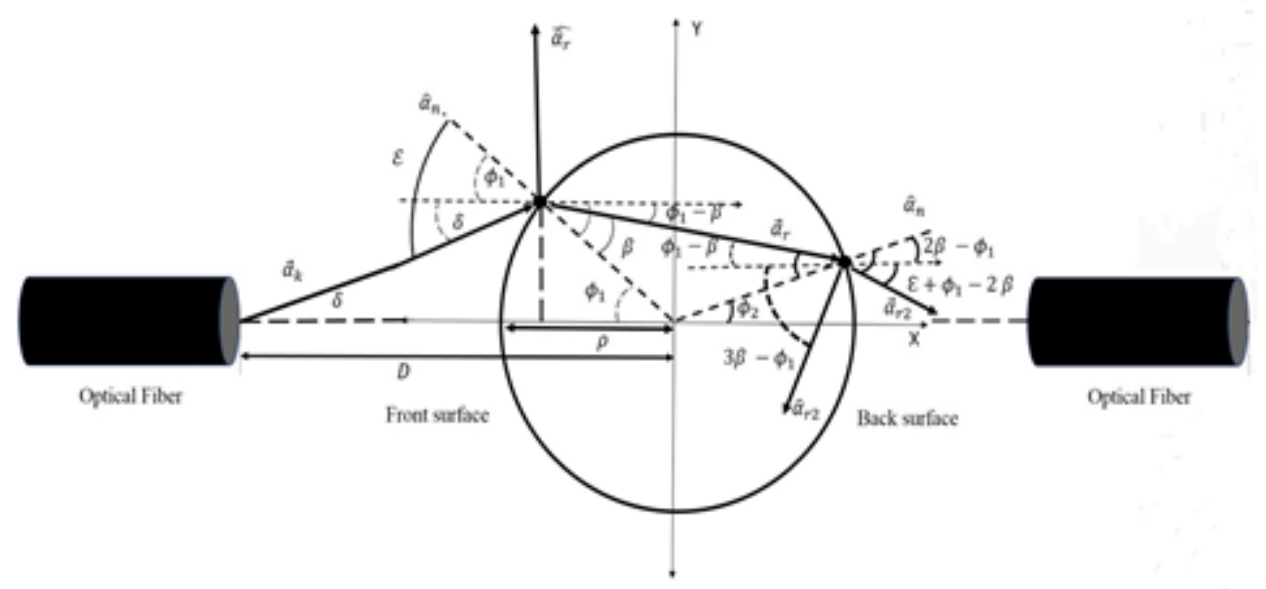

Figure 2: Representation of incident, reflected and transmitted rays on a spherical object trapped in the path of two counter-propagating beams. Note that for simplicity the rays are shown only for one beam.

\subsection{Experimental setup}

Figure 3A shows a schematic of our dual-beam setup. Figure 3B shows the dual-beam optical set up used in our trapping and stretching experiments. This setup utilizes two multi-axis positioning stages to position the optical fibers directly counter-propagating from one another 
with $<5 \mu \mathrm{m}$ accuracy in the $\mathrm{XY}$ plane as seen under the $\mathrm{KH} 1300$ Microscope. Two high power laser sources (975 $\mathrm{nm}$ wavelength) were used to derive the optical beams through each fiber. An optical power meter with an accompanying photodetector was used to measure the output power of a cleaved and polished optical fibers before use. The sample stage along with fiber ends was observable on big screen through a digital microscope image acquisition.
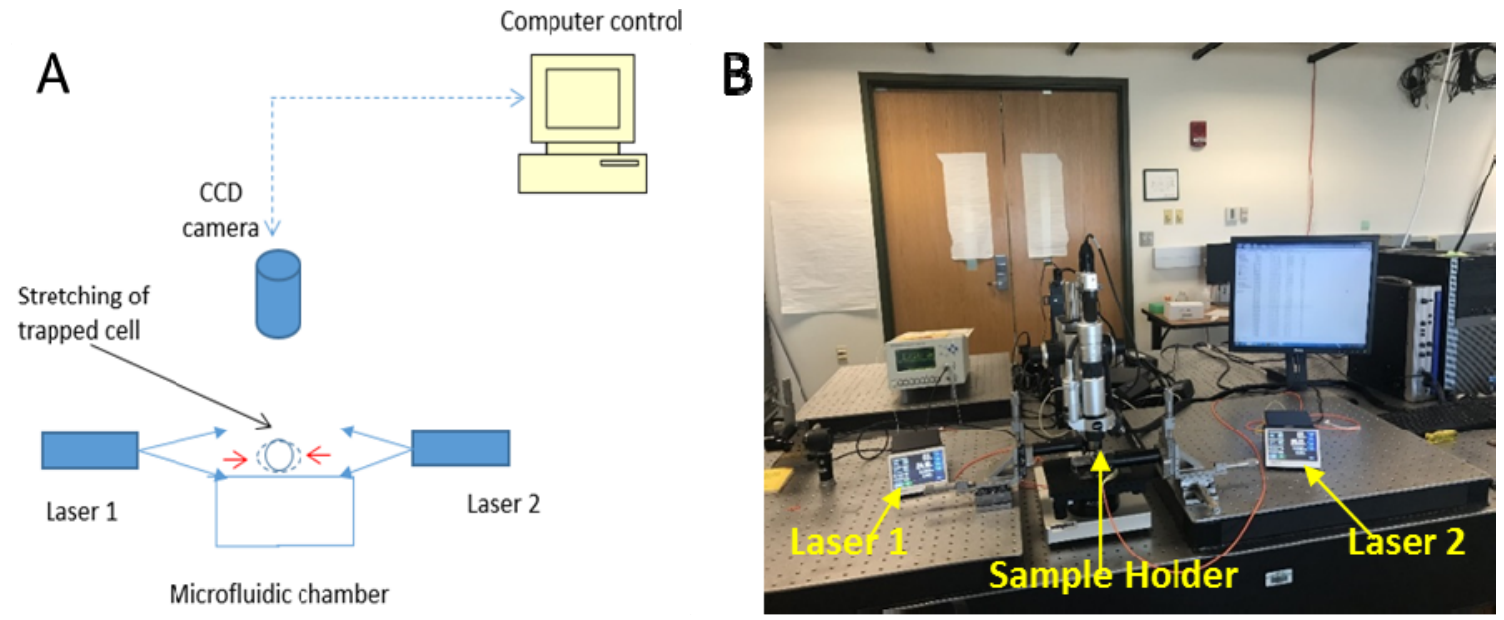

Figure 3: Dual-beam experimental setup used in our experiments.

2.3 HEK cell stretching: Human embryonic kidney 293 (HEK293) cells were purchased from American Type Culture Collection (ATCC; Manassas, VA). They were grown in cell culture flasks following the supplier's protocol. Briefly, the frozen cells were thawed at $37{ }^{\circ} \mathrm{C}$ water bath, transferred to $10 \mathrm{ml}$ tube containing fresh growth medium (see below) and centrifuged at $1000 \mathrm{rpm}$. The cell pellet was resuspended in Eagle's Minimum Essential Medium (EMEM), containing L-glutamine and 10\% FBS and transferred to the cell culture flask in a sterile hood. The culture was transferred to a $37{ }^{\circ} \mathrm{C}$ incubator that was maintained with $5 \% \mathrm{CO} 2$ until the flask reached $80 \%$ confluence. The cells were harvested and resuspended in Hanks' balanced salt solution (HBSS; Atlanta Biologicals, Lawrenceville, GA) and used for the stretching experiments.

The cells suspended in HBSS were dropped in the sample holding area between the two optical fibers. The cells were trapped using the two counter-propagating, diffraction-limited diverging beams at various trapping powers. The trapping power was expressed as total optical power from both the beams. For each power, the cell was maintained in the trap for 30 seconds to allow for adequate time for its membrane deformation. Continuous images were acquired during the entire 30 -sec protocol using the digital microscope at the frame rate of 15 frames per second.

2.4 Deformation analysis: The acquired images were processed in MATLAB to determine the cell deformation using image processing toolbox. Image thresholding based on grayscales was 
used to delineate the cell boundaries in the acquired images. In order to compensate for the errors due to cell motion in the z-axis within the trap, an aspect ratio (ratio of the most stretched axis to the least stretched axis) of the cell was calculated to determine the cell deformation, similar to Guck et al. [5].

\section{EXPERIMENT RESULTS}

\subsection{Stretching of HEK cells}

We successfully trapped and stretched HEK cells using the above described dual-beam OS setup (Se Figure 4A. The minimum total trapping power needed for HEK cells was $80.5 \mathrm{~mW}$. However, this power was not sufficient to stretch the cell. Figure 4 shows the delineated boundaries of the original HEK cell just prior to entering the trap (Panel B) and the same cell at the end of $30 \mathrm{sec}$ trap (Panel C) under the total power of $164 \mathrm{~mW}$. The delineated cell boundary in Panel B clearly shows cell deformation along the axis of light path.
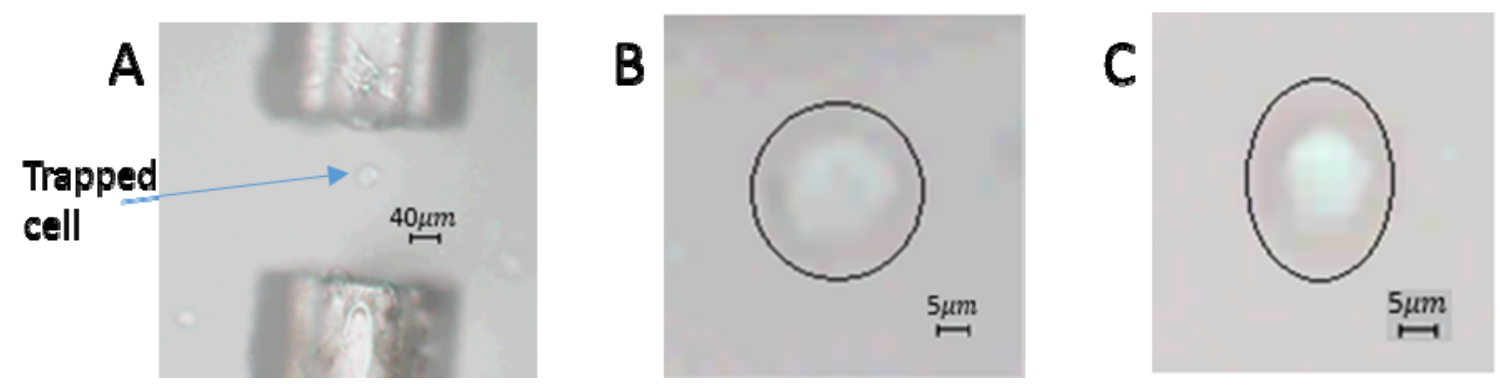

Figure 4: A) Trapped HEK cell between two optical fibers carrying counter-propagating laser beams. Delineated cell boundary B) before and C) after 30 seconds in the trap under 164

Figure 5 shows cell deformations of HEK cells for varying trapping powers $(n=5$ for each power). The powers used to trap and stretch cells were $100 \mathrm{~mW}, 164 \mathrm{~mW}, 329 \mathrm{~mW}, 425 \mathrm{~mW}$ and $561 \mathrm{~mW}$. The figure clearly shows that the extent of deformation increases as power increases (16.7\% at $100 \mathrm{~mW}$ to $40 \%$ at $561 \mathrm{~mW}$ ). 


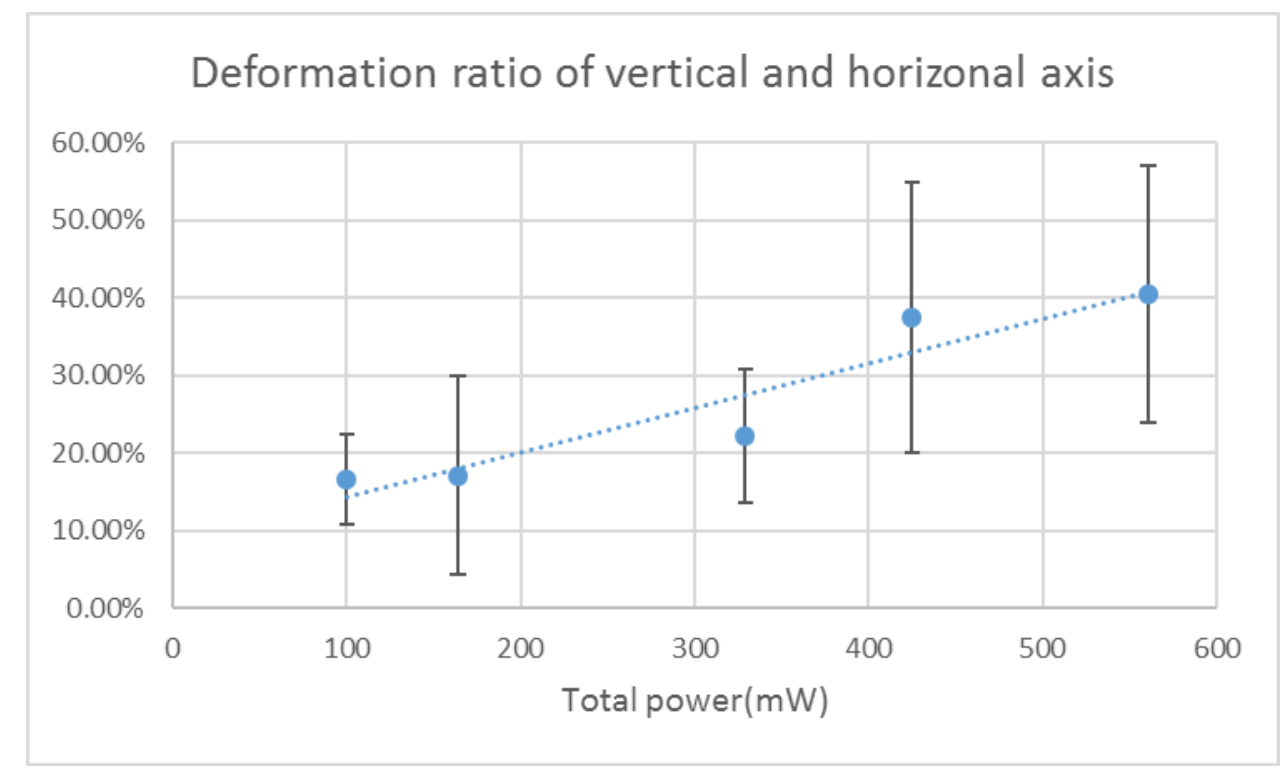

Figure 5: Plot of the cell deformation with increasing power ( $n=5$ for each power).

\subsection{Stretching of Cancer cells}

We also attempted to trap MBC cells using the same setup (Figure 6) in order to study the differences in cell deformation ability of the two cell types. The minimum trapping power for MBC cells was significantly lower $(65.2 \mathrm{~mW})$ as compared to the HEK cells under the same conditions. Figure 6 shows a representative example of a breast cancer cell before trapping (Panel B) and 30 seconds post-trapping (Panel C) under the total power of $80 \mathrm{~mW}$. The image analysis determined the deformation ratio in MBC cells as $32.5 \%(n=2)$ which is significantly higher to that of in HEK cell for the same trapping power.
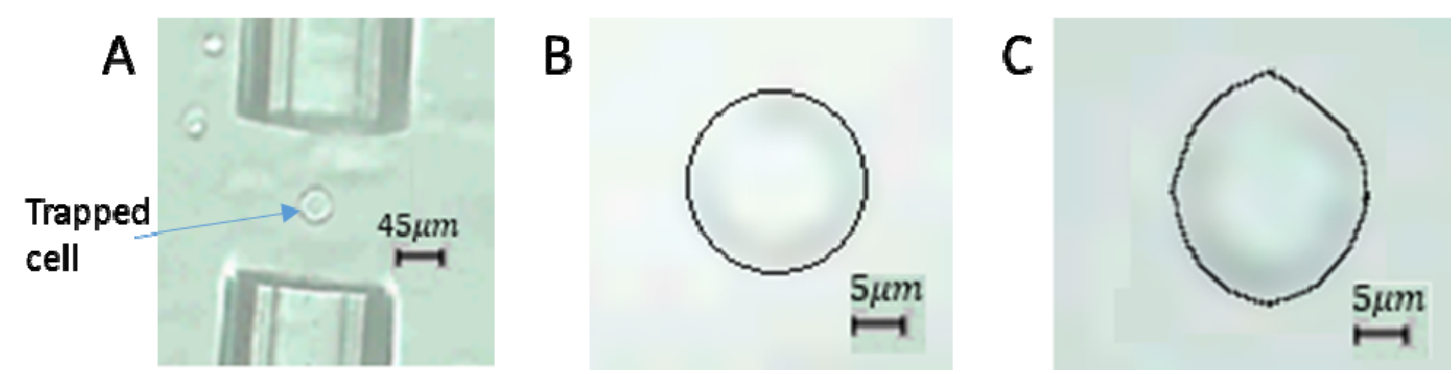

Figure 6: A) Trapped MBC cell between two optical fibers carrying counter-propagating laser beams. Delineated cell boundary B) before and C) after 30 seconds in the trap under $80 \mathrm{~mW}$

\section{CONCLUSIONS}

In this study, we utilized a dual-beam optical setup to successfully trap and stretch HEK and MBC cells without any mechanical contact. The minimum optical power needed to trap MBC 
cells was significantly lower $(65.2 \mathrm{~mW})$ to that of HEK cells $(80.5 \mathrm{~mW})$. We were able to achieve $40 \%$ cell stretching in HEK cells and $32.5 \%$ in MBC cells. It was observed that the percentage deformation in cells monotonically increased with the increase in optical power of the trap. This study provides useful insights into the characterization of cytoskeletal elasticity in the two cell types based on non-contact optical cell stretching. More experiments are warranted to establish a definitive trend in cell deformation in the two cell types, which could be useful in identifying early onset of disease based on the mechanical characterization of cells.

\section{Funding Acknowledgement}

This work was supported by the National Science Foundation grant 1647800.

\section{References:}

[1] B. Lincoln et al., "Reconfigurable microfluidic integration of a dual-beam laser trap with biomedical applications," Biomed. Microdevices, vol. 9, no. 5, pp. 703-710, 2007.

[2] A. Ashkin, "Acceleration and Trapping of Particles by Radiation Pressure," Phys. Rev. Lett., vol. 24, no. 4, pp. 156-159, Jan. 1970.

[3] Guck, Jochen et al. "The Optical Stretcher: A Novel Laser Tool to Micromanipulate Cells", Biophysical Journal 81, 767-784 (2001).

[4] Tie Yang, Francesca Bragheri and Paolo Minzioni" A Comprehensive Review of Optical Stretcher for Cell Mechanical Characterization at Single-Cell Level". May 2016

[5] Guck, Jochen et al. "Optical Deformability as an Inherent Cell Marker for Testing Malignant Transformation and Metastatic Competence ". Biophys. J. Lett. 88, 3689 (2005).

[6] R. M. Hochmuth, "Micropipette aspiration of living cells," J. Biomech., vol. 33, no. 1, pp. 15-22, 2000.

[7] O. Thoumine, A. Ott, O. Cardoso, and J. J. Meister, "Microplates: a new tool for manipulation and mechanical perturbation of individual cells," J. Biochem. Biophys. Methods, vol. 39, no. 1-2, pp. 47-62, Feb. 1999.

[8] T. G. Kuznetsova, M. N. Starodubtseva, N. I. Yegorenkov, S. A. Chizhik, and R. I. Zhdanov, "Atomic force microscopy probing of cell elasticity," Micron, vol. 38, no. 8, pp. 824833, 2007. 
[9] Hulst, HC Van De. Light Scattering by Small Particles. NY, John Wiley \& Sons, 1957, 172-176.

[10] P. B. Bareil, Y. Sheng and A. Chiou, "Local stress distribution on the surface of a spherical cell in an optical stretcher," Opt. Express 14, 12503-12509 (2006).

[11] Bareil, Paul B., Yunlong Sheng, Yin-Quan Chen, and Arthur Chiou. "Calculation of Spherical Red Blood Cell Deformation in a Dual-beam Optical Stretcher." Optics Express 15 (2007).

[12] Sciencell Research Laboratories, Hank's Balanced Salt Solution, https://www.sciencellonline.com/hank-s-balanced-salt-solution.html 